\title{
Platelet-derived growth factor-D promotes colorectal cancer cell migration, invasion and proliferation by regulating Notch1 and matrix metalloproteinase-9
}

\author{
BIN JIANG ${ }^{*}$, JINHUANG CHEN**, WENZHENG YUAN, JINTONG JI, \\ ZHENGYI LIU, LIANG WU, QIANG TANG and XIAOGANG SHU \\ Department of Gastrointestinal Surgery, Union Hospital, Tongji Medical College, \\ Huazhong University of Science and Technology, Wuhan, Hubei 430022, P.R. China
}

Received January 13, 2016; Accepted June 2, 2017

DOI: $10.3892 / \mathrm{ol} .2017 .7510$

\begin{abstract}
Colorectal cancer (CRC) has been one of the most common types of cancer for decades worldwide. The pathogenesis of CRC is associated with the processes of activating oncogenes and inactivating anti-oncogenes. Platelet-derived growth factor-D (PDGF-D) was confirmed to regulate migration, invasion, proliferation, apoptosis and metastasis in various cancer cells. Overexpression of PDGF-D exists in a number of human malignancies, including pancreatic, prostate and breast cancer. However, the expression and function of PDGF-D and its associated molecular mechanism in CRC remain unclear. Thus, the expression of PDGF-D was detected in CRC tissues and human colon cancer lines. Subsequently, the effects of PDGF-D on the invasion, migration and proliferation of cancer cells were investigated. The corresponding molecular mechanism had also been explored. The present study revealed that PDGF-D was upregulated not only in CRC tissues but also in CRC cell lines, and simultaneously, facilitated the processes of migration, invasion and proliferation. Silencing PDGF-D in the SW480 cell line inhibited migration, invasion and proliferation distinctly, with reduced expression of Notch1 and matrix metalloproteinase-9. Furthermore, upregulating PDGF-D in HCT116 cells led to the opposite results. These findings indicate that PDGF-D may be developed into a potential therapeutic target for CRC treatment.
\end{abstract}

Correspondence to: Professor Xiaogang Shu, Department of Gastrointestinal Surgery, Union Hospital, Tongji Medical College, Huazhong University of Science and Technology, 1277 Jiefang Avenue, Wuhan, Hubei 430022, P.R. China

E-mail:sxg678@yahoo.com

${ }^{*}$ Contributed equally

Abbreviations: CRC, colorectal cancer; PDGF-D, platelet-derived growth factor-D; PDGFR- $\beta$, platelet-derived growth factor receptor- $\beta$

Key words: platelet-derived growth factor-D, colorectal cancer, migration, invasion, proliferation, Notch1, matrix metalloproteinase-9

\section{Introduction}

Colorectal cancer (CRC) is one of the most common types of malignancy in Western countries, and is the cause of a high number of cancer-associated mortalities every year (1). In the past decades, the prevalence of CRC in China has increased rapidly, which may be associated with westernized lifestyle, longer lifespan and a poor CRC screening system $(2,3)$. Previous studies have shown that the process of CRC formation refers to a gradual activation of oncogenes and inactivation of anti-oncogenes (4). However, the mechanisms of CRC tumorigenesis and tumor progression are still not illustrated clearly. Consequently, identifying more credible biomarkers may help with CRC prognosis estimation and provide novel potential targets for therapy.

Platelet-derived growth factor (PDGF)-D belongs to the PDGF family, whose members are known as a mesenchymal growth factors that promote epithelial-stromal communication (5). Platelet-derived growth factor-D (PDGF-D) exerts its biological functions by specifically binding to its cognate receptor, platelet-derived growth factor receptor- $\beta$ (PDGFR- $\beta$ ), leading to rapid phosphorylation of PDGFR- $\beta$ and consequent activation of numerous downstream signaling pathways (6). Previous studies have shown that PDGF-D is involved in carcinogenesis, particularly regulating the course of epithelial-to-mesenchymal transition, which facilitates tumor proliferation and metastasis (7-9). Furthermore, PDGF-D has been reported to be associated with the mechanistic target of rapamycin (mTOR), Notch, nuclear factor- $\kappa \mathrm{B}(\mathrm{NF}-\kappa \mathrm{B}), \mathrm{B}-\mathrm{cell}$ lymphoma-2 (Bcl-2) and $\mathrm{CXC}$ motif chemokine receptor 4 (CXCR4) signaling pathways (10). Overexpression of PDGF-D was also observed in various human tumors, including pancreatic, prostate, gastric and breast cancer, predicting that PDGF-D is involved in cancer development and progression (7-9,11).

As there is little clear evidence about the expression and function of PDGF-D in CRC thus far, it will be necessary to explore the significant role of PDGF-D in CRC. In the present study, PDGF-D messenger RNA (mRNA) level and protein expression were examined in CRC tissues, paired normal tissues and cell lines. The effects of PDGF-D on CRC 
behaviors, including migration, invasion and proliferation, were then surveyed. By upregulating and downregulating the expression level of PDGF-D in colon cell lines, the detailed functions of PDGF-D were studied, with the aim of clarifying its potential mechanism.

\section{Materials and methods}

Ethics statement and patient tissue samples. The present study was approved by the Ethics Committee of Tongji Medical College, Huazhong University of Science and Technology (Wuhan, China). A total of $45 \mathrm{CRC}$ tissue samples were collected from patients with CRC who had received surgical treatment in the Union Hospital affiliated to Huazhong University of Science and Technology between September 2014 and August 2015. The present study included 19 and 26 male and female patients, respectively (mean age, 52.82 years; age range, 25-87 years). All samples were obtained with written informed consent from the patients. Part of these specimens were immersed in $5 \%$ paraformaldehyde solution at $4^{\circ} \mathrm{C}$ for immunohistochemistry (IHC), and the remaining were immediately frozen and stored at $-80^{\circ} \mathrm{C}$.

IHC. CRC specimens were fixed in 5\% paraformaldehyde solution, as described previously (12). For calculating the protein level of PDGF-D, the general procedure was designed as follows: i) Record the intensity of dyeing in stained tissues (negative, 0 ; weak positive, 1 ; moderate positive, 2; and strong positive, 3); ii) calculate the quantity of stained cancer cells $(0,<10 \% ; 1,<25 \% ; 2,<50 \%$; and $3, \geq 50 \%)$; iii) multiply the two indexes to calculate the staining index (SI); and iv) the final expression is considered negative when $\mathrm{SI}<3$ and positive when $\mathrm{SI} \geq 4$.

Cell culture and lentivirus transfection. Human colon cancer SW480 and SW620 cells were cultured in L15 (HyClone; GE Healthcare Life Sciences, Little Chalfont, UK), HCT116 cells were cultured in McCoy's 5A (HyClone; GE Healthcare Life Sciences), DLD-1 cells were cultured in RPMI-1640 (Wuhan Goodbio Technology, Wuhan, China) and LoVo cells were cultured in DMEM (Gibco; Thermo Fisher Scientific, Inc., Waltham, MA, USA) complete medium with $10 \%$ fetal bovine serum (ScienCell Research Laboratories, Inc., Carlsbad, CA, USA), and were grown in $25-\mathrm{cm}^{2}$ polystyrene tissue culture flasks (Corning Incorporated, Corning, NY, USA) at $37^{\circ} \mathrm{C}$ in an atmosphere containing $5 \% \mathrm{CO}_{2}$ for between 48 and $72 \mathrm{~h}$. Lentivirus transfection was used to up- and downregulate the PDGF-D expression in cell lines. The specific procedure has been described previously (13). The short hairpin (sh)PDGF-D-lentivirus and complementary DNA (cDNA)-PDGF-D-lentivirus were purchased from Shanghai GeneChem., Shanghai, China.

Detection of apoptosis by flow cytometry. Subsequent to successful transfection, the SW480 and HCT116 cells were collected to conduct detection of apoptosis by flow cytometry, and the specific operation steps have been presented in a previous study (14). The Annexin V/propidium iodide apoptosis detection kit was used according to the manufacturer's protocol (Wuhan Antgene Biotechnology, Wuhan, China).
Reverse transcription-quantitative polymerase chain reaction (RT-qPCR). The detailed procedures and notes of RT-qPCR were reported in a previous study (15). In brief, TRIzol reagent (Invitrogen; Thermo Fisher Scientific, Inc.) was used to extract total RNA from cells, CRC tissues and paired normal controls. Subsequently, the RT of total RNAs was performed with PrimeScript RT Master mix (Takara Bio, Inc., Otsu, Japan). Finally, the expected nucleic acid amplification products were detected in the StepOnePlusÔ Real-Time PCR system (Applied Biosystems; Thermo Fisher Scientific, Inc.) with SYBR-Green Master mix (Takara Bio, Inc.). mRNA quantity of each gene was calculated using the $2^{-\Delta \Delta \mathrm{Cq}}$ method (16) and normalized to GAPDH. The primer sequences of PDGF-D (forward, GTG GAGGAAATTGTGGCTGT and reverse, CGTTCATGGTGA TCCAACTG), and the internal control (GAPDH forward, GGGGAGCCAAAAGGGTCATCATCT and reverse, GAC GCCTGCTTCACCACCTTCTTG) were designed according to a previous study (17).

Western blot analysis. The specimens and cells were lysed in an appropriate amount $(100 \mu \mathrm{l})$ of lysis buffer (Beyotime Institute of Biotechnology, Haimen, China) with phenylmethylsulfonyl fluoride for total protein extraction. The protein concentration in the lysates was then detected by bicinchoninic acid assay. Equal quantities of total protein (10 $\mu \mathrm{l} /$ lane) were loaded onto SDS-PAGE (12\% gel) and then separated by electrophoresis. The separated proteins were transferred to a polyvinylidene fluoride membrane, which was subsequently blocked with 5\% skimmed milk and TBS-Tween-20 for $1 \mathrm{~h}$ at room temperature. The membranes were then incubated with primary antibodies at $4^{\circ} \mathrm{C}$ overnight. The primary antibodies used in the study were as follows: Anti-PDGF-D (cat. no. ab181845; dilution, 1:500; Abcam, Cambridge, MA, USA), anti-PDGFR- $\beta$ (cat. no. Esap11645; dilution, 1:500; Wuhan Elabscience Biotechnology, Wuhan, China), anti-Notch1 (cat. no. SC-6014; dilution, 1:500; Santa Cruz Biotechnology, Inc., Dallas, TX, USA) and anti-MMP-9 (cat. no. ab58803; dilution, 1:500; Abcam). Subsequently, either the horseradish peroxidase (HRP-) labeled goat anti-rabbit immunoglobulin G (cat. no. GB23303; dilution, 1:1,000; Wuhan Goodbio Technology) or the HRP-labeled goat anti-mouse IgG (cat. no. GB23301; dilution, 1:1,000; Wuhan Goodbio Technology) secondary antibody was applied to the membrane for $1 \mathrm{~h}$ at room temperature. An enhanced chemiluminescence system (cat. no. G2014; Wuhan Goodbio Technology) and Image J software (version 2; National Institutes of Health, Bethesda, MD, USA) were used to analyze the protein expression.

Transwell migration and Matrigel invasion assays. Cell Transwell assays were performed to test the invasion and migration of cancer cells. The specific procedure was previously reported (18). In the present study, Transwell chambers (24-well, 8- $\mu$ m pore membranes) were purchased from Corning Incorporated. Matrigel (Sigma-Aldrich; Merck KGaA, Darmstadt, Germany) was used in cell invasion analysis, but not in the migration assay.

Cell proliferation assays. A Cell Counting kit (CCK)-8 assay (Beyotime Institute of Biotechnology) was used to detect cell proliferation. Following inoculation onto a 96-well plate, cells 
A

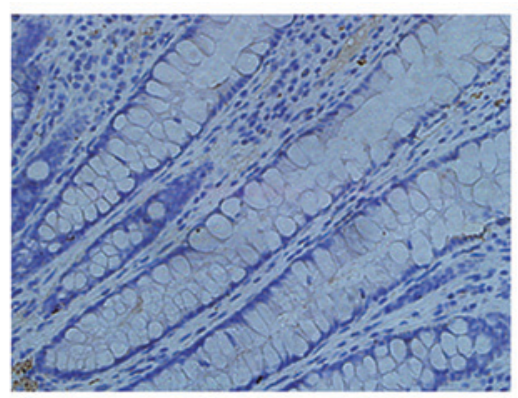

C

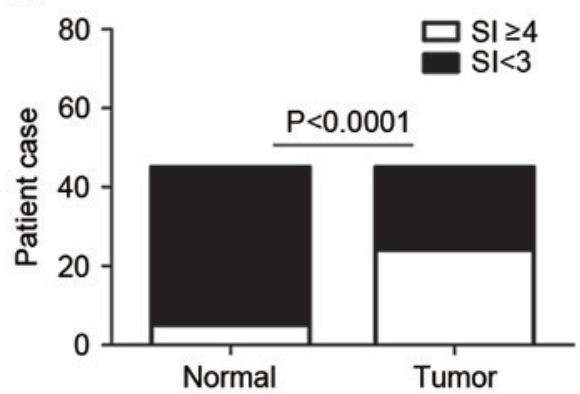

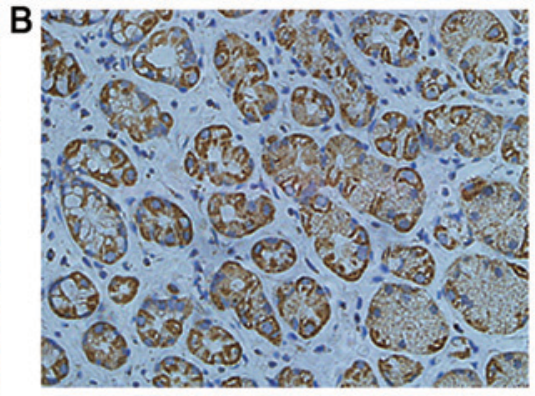

D

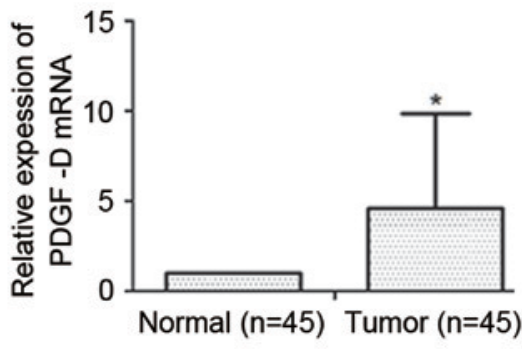

Figure 1. PDGF-D is highly expressed in CRC tissues. (A) Representative immunostaining of PDGF-D in adjacent noncancerous tissues (original magnification, x200). (B) Representative immunostaining of PDGF-D in CRC (original magnification, x200). (C) Statistical analysis of the expression of PDGF-D protein in patients with CRC. (D) The mRNA level of PDGF-D in CRC tissues was significantly higher than that in paired adjacent normal tissues by quantitative polymerase chain reaction. " $\mathrm{P}<0.05$. PDGF-D, platelet-derived growth factor-D; CRC, colorectal cancer; SI, staining index; mRNA, messenger RNA.

were cultured in an incubator for $24 \mathrm{~h}$ at $37^{\circ} \mathrm{C}$ in an atmosphere containing $5 \% \mathrm{CO}_{2}$. An appropriate amount $(10 \mu \mathrm{l})$ of CCK-8 solution was then added to each well, and subsequently, the cells were kept in the incubator for 1-4 h. Eventually, cell proliferation was analyzed by measuring the absorbance at $450 \mathrm{~nm}$ with a microplate reader (Thermo Fisher Scientific, Inc.).

Statistical analysis. All experiments were performed at least three times independently. All results shown in our study were analyzed using SPSS statistical software (version 22.0; IBM Corp., Armonk, NY, USA). The results are expressed as the mean \pm standard deviation. Statistical analyses were performed using Student's $t$ and $\chi^{2}$ tests. $\mathrm{P}<0.05$ was considered to indicate a statistically significant difference.

\section{Results}

PDGF-D is highly expressed in CRC tissues. PDGF-D protein expression was investigated through IHC detection, which revealed that PDGF-D was highly expressed in CRC tissues (Fig. 1A and B). In total, 53.3\% (24/45) of CRC tissues were positive for PDGF-D protein expression in the cytoplasm, while $11.1 \%(5 / 45)$ of the paired normal colorectal tissues were positive for PDGF-D protein expression $(\mathrm{P}<0.0001$; Fig. 1C). Subsequently, the mRNA level of PDGF-D was detected in CRC tissues and paired normal colorectal tissues by qPCR analysis. The results demonstrated that PDGF-D mRNA expression was averagely 4.6-fold higher in CRC tissues than in normal tissues $(\mathrm{P}<0.05$; Fig. 1D).

PDGF-D and PDGFR are expressed at a high level in the SW480 cell line and a low level in HCT116 cells. Several colon cell lines were screened for the expression of PDGF-D, including
SW480, SW620, HCT116, DLD-1 and LoVo cells (data of SW620, DLD-1 and LoVo cells are not shown). Finally, SW480 was selected for its stable high expression of PDGF-D protein and HCT116 was selected for its relatively low expression of PDGF-D (Fig. 2A and B). The corresponding mRNA levels were detected by qPCR and the results were similar to the expression of PDGF-D protein (Fig. 2C). Furthermore, the expression of PDGFR- $\beta$ in SW480 and HCT116 cell lines was detected by qPCR and western blot analysis. The results demonstrated that PDGFR- $\beta$ was highly expressed in SW480 cells and expressed at a lower level in HCT116 cells (Fig. 2).

Knockdown of PDGF-D in SW480 cells decreases the migration, invasion and proliferation capacity of cancer cells by downregulation of Notch1 and MMP-9. Knockdown of PDGF-D in the SW480 cell line was successfully established by lentiviral transfection (Fig. 3A). The capacity of migration, invasion and proliferation of SW480 cells was significantly decreased compared with that of the control (Fig. 3B-D). In order to study the potential mechanism, the associated signaling pathways reported previously, including mTOR, Notch, NF-кB, Bcl-2 and CXCR4, were screened by western blot analysis (10). The expression of Notch1 and MMP-9 was observed to decline following knockdown of PDGF-D in SW480 cells (Fig. 3A). Furthermore, the apoptotic rate of transfected SW480 cells was determined. No clear difference was observed between the shPDGF-D-lentivirus-transfected group and the negative control group (Fig. 3E and F; negative control vs. shPDGF-D-lentivirus $=6.5$ vs. $6.8 \%$ ).

Upregulation of PDGF-D expression in HCT116 cells enhances the capacity of migration, invasion and proliferation of cancer cells by increasing Notchl and MMP-9 levels. Upregulation of PDGF-D expression in the HCT116 

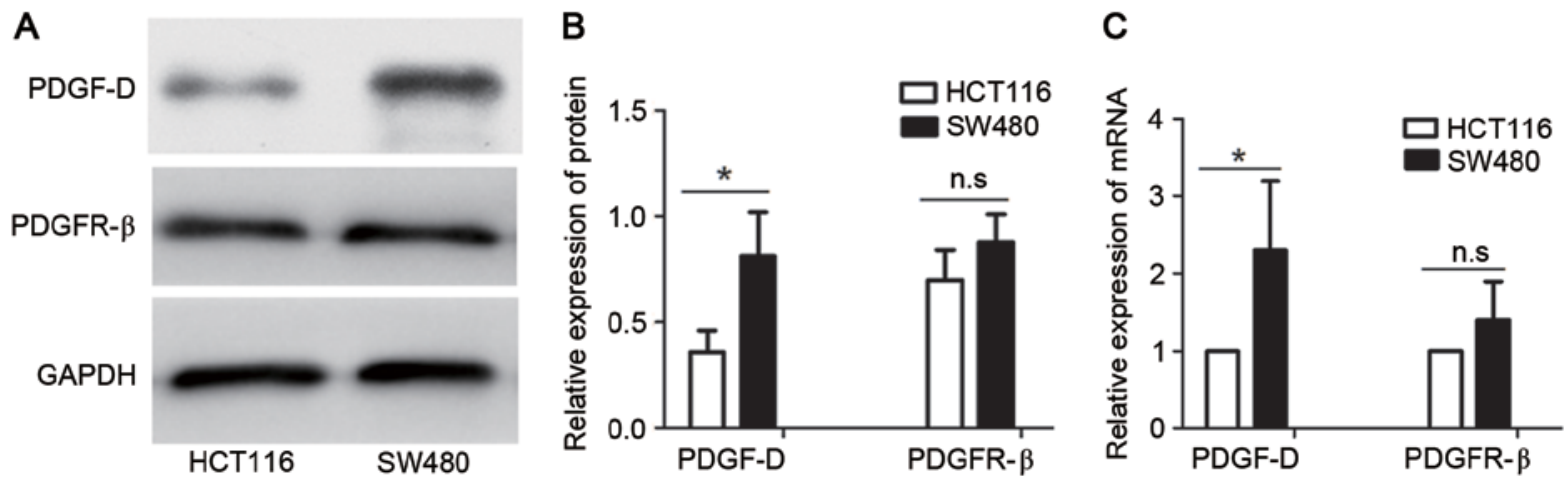

Figure 2. PDGF-D and PDGFR- $\beta$ are highly expressed in the SW480 cell line, and expressed at low levels in the HCT116 cell line. (A) The expression of PDGF-D and PDGFR- $\beta$ protein in SW480 cells and HCT116 cells was analyzed by western blotting. (B) Satistical analysis of the expression of PDGF-D and PDGFR- $\beta$ protein in SW480 and HCT116 cells. (C) The mRNA levels of PDGF-D and PDGFR- $\beta$ were analyzed by quantitative polymerase chain reaction. ${ }^{*} \mathrm{P}<0.05$. n.s, non-significant; PDGFR, platelet-derived growth factor receptor; mRNA, messenger RNA.
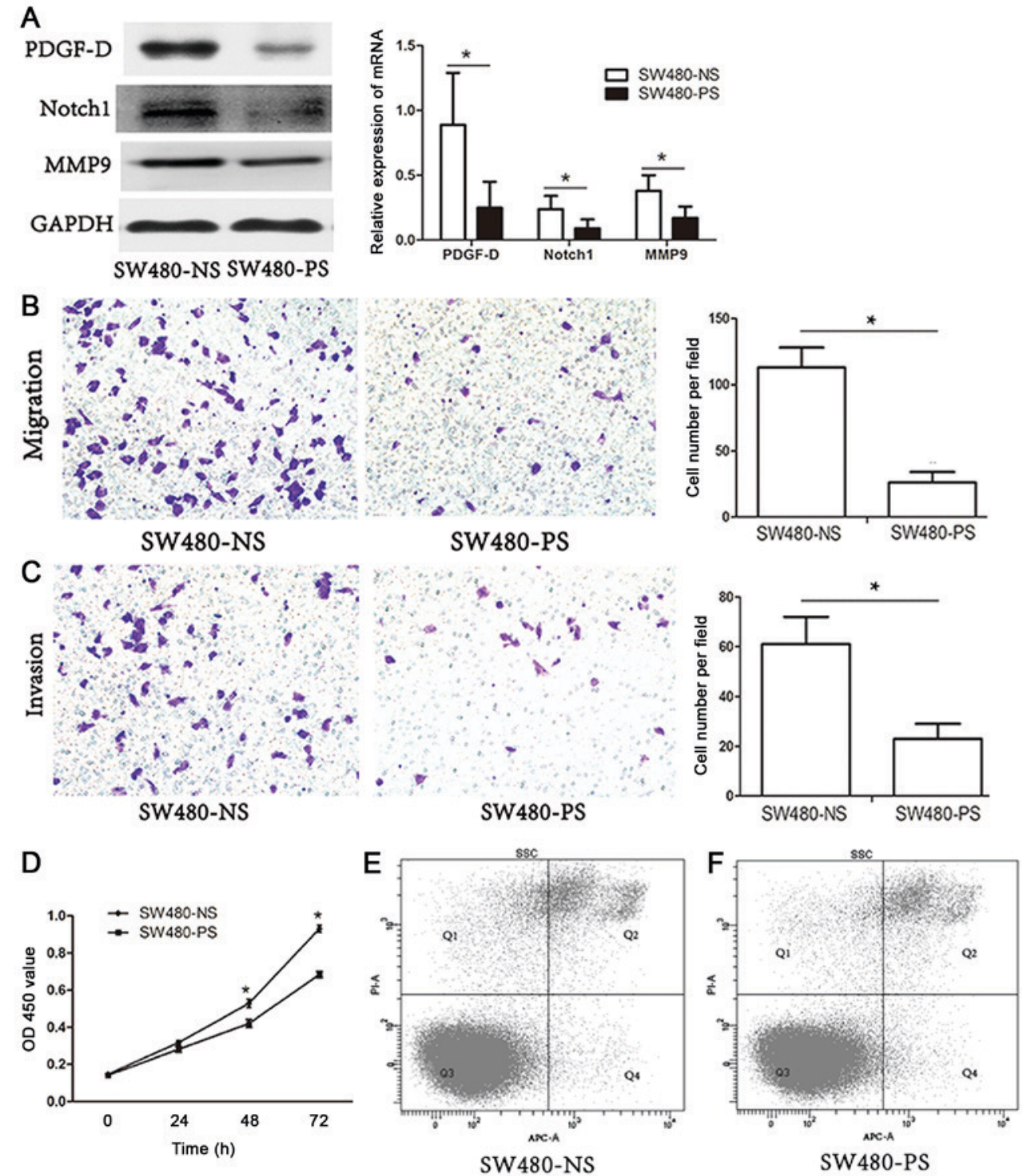

Figure 3. Knockdown of PDGF-D in SW480 cells declines the migration, invasion and proliferation capacity of cancer cells by downregulation of Notch1 and MMP-9. (A) Expression of PDGF-D, Notch1 and MMP-9 in SW480 cells infected with shPDGF-D-lentivirus and negative control by western blot analysis. (B) SW480 cells were transfected with shPDGF-D-lentivirus or negative control for $48 \mathrm{~h}$, and a Transwell migration assay was then performed. (C) SW480 cells were transfected with shPDGF-D-lentivirus or negative control for $48 \mathrm{~h}$, and then used for Transwell invasion assay. (D) SW480 cells were transfected with shPDGF-D-lentivirus or negative control for $48 \mathrm{~h}$, and a Cell Counting kit-8 assay was then performed. (E) The apoptotic rate of the negative control group was $6.5 \%$ by Annexin V/PI staining. (F) The apoptotic rate of the shPDGF-D-lentivirus group was $6.8 \%$ by Annexin V/PI staining. "P<0.05. NS, non-specific shRNA; PS, PDGF-D shRNA; PDGF-D, platelet-derived growth factor-D; MMP-9, matrix metalloproteinase-9; sh, short hairpin; PI, propidium iodide; OD, optical density; mRNA, messenger RNA; Q, quadrant; APC, allophycocyanin; SSC, side scatter. 
A
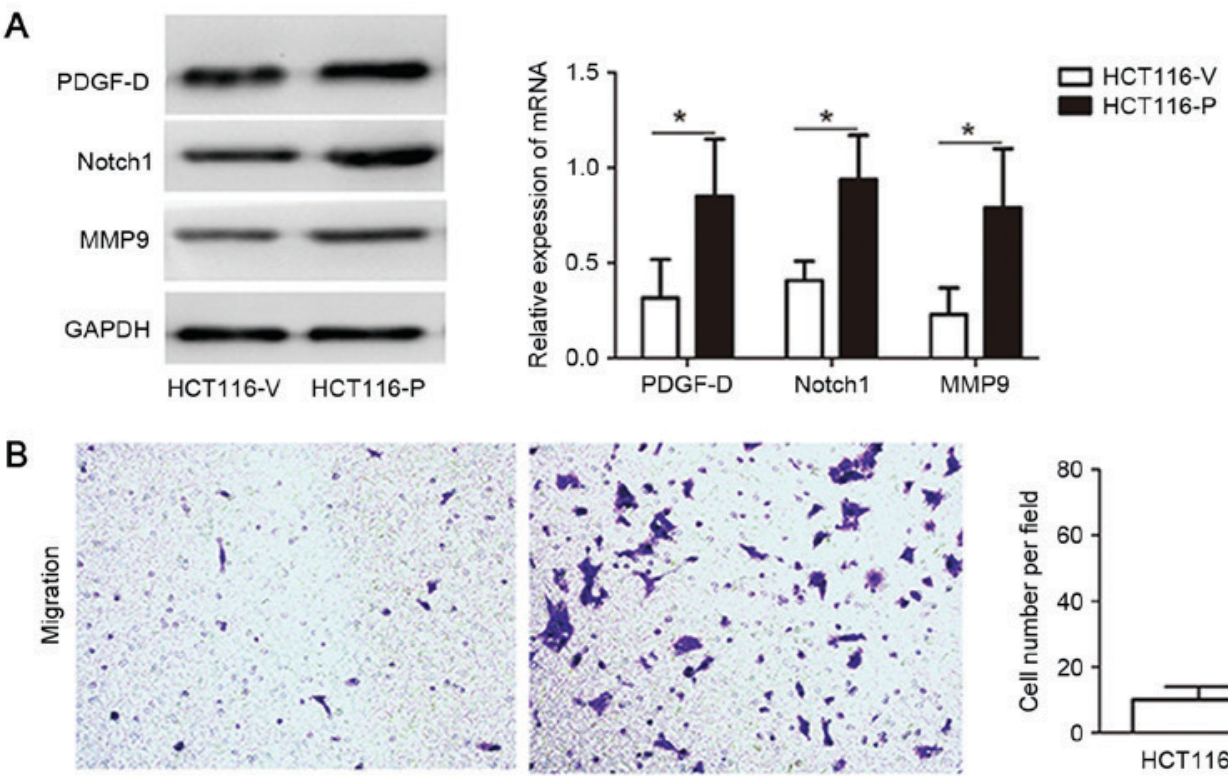

HCT116-V

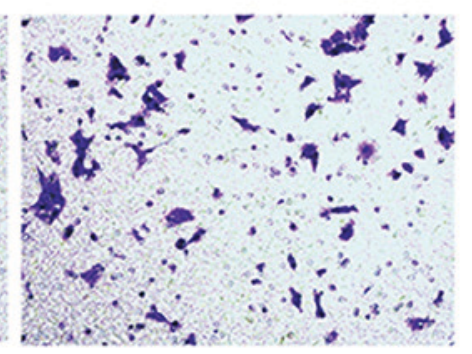

HCT116-P
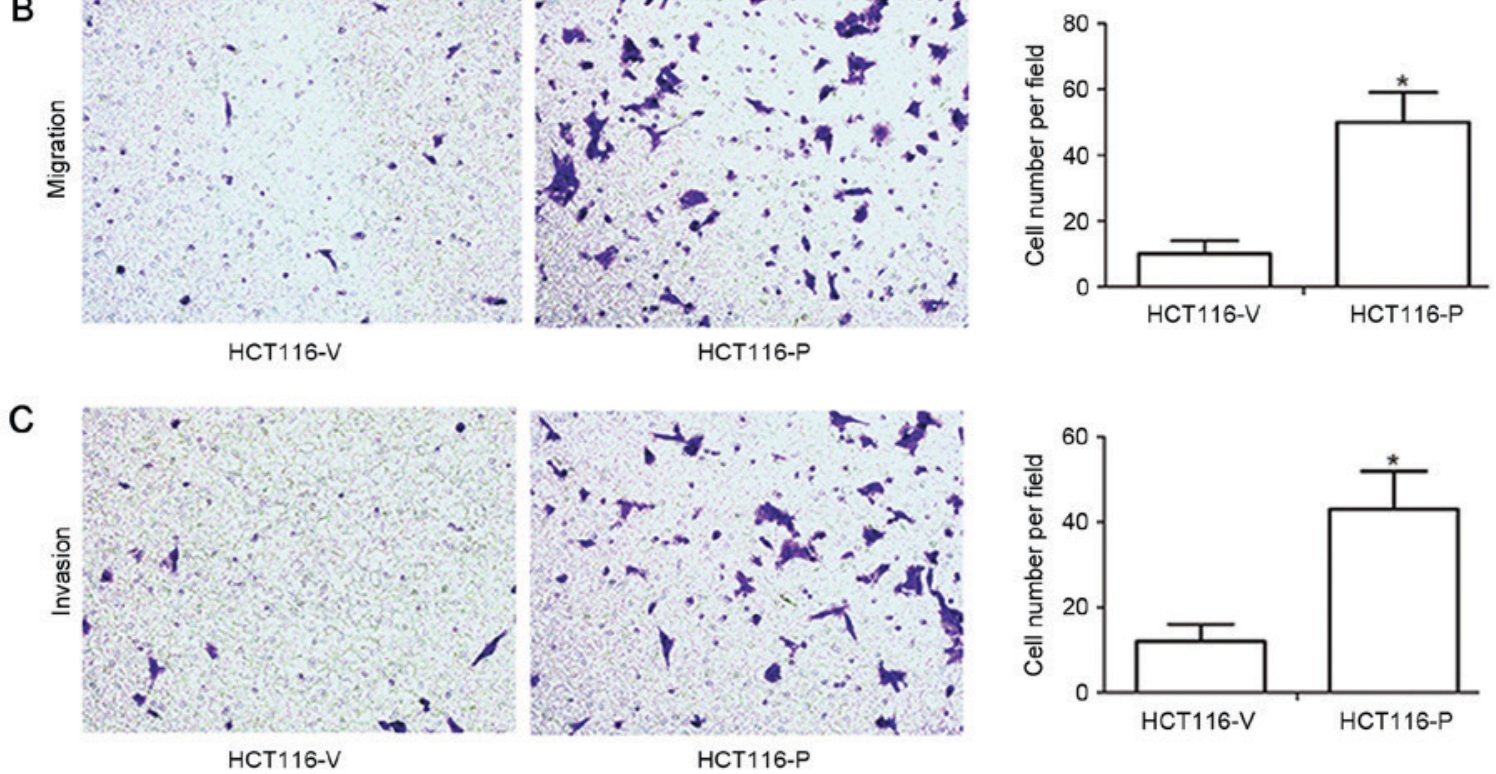

D

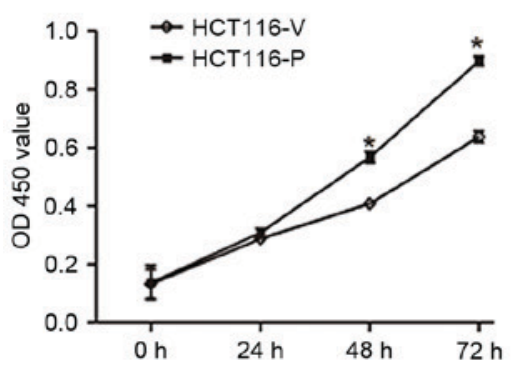

$\mathrm{E}$

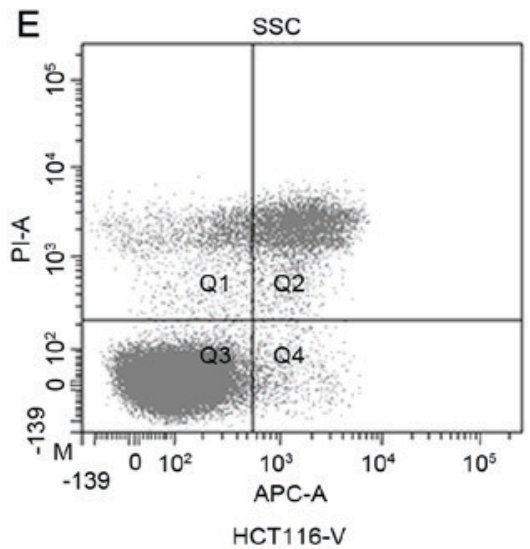

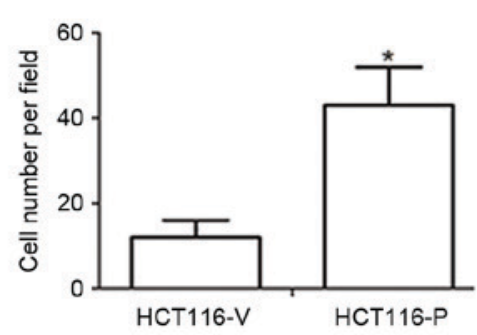

$\mathrm{F}$

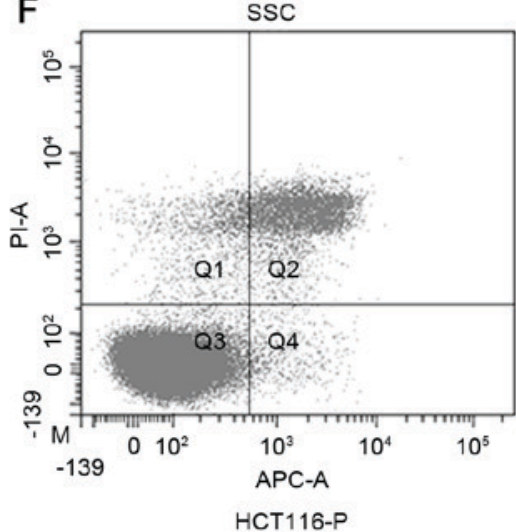

Figure 4. Upregulation of PDGF-D in the HCT116 cell line enhances the capacity of migration, invasion and proliferation of cancer cells by increasing Notch1 and MMP-9 expression. (A) Expression of PDGF-D, Notch1 and MMP-9 in HCT116 cells infected with cDNA-PDGF-D-lentivirus and negative control by western blot analysis. (B) HCT116 cells were transfected with cDNA-PDGF-D-lentivirus or negative control for $48 \mathrm{~h}$, and then used for Transwell migration assay. (C) HCT116 cells were transfected with cDNA-PDGF-D-lentivirus or negative control for $48 \mathrm{~h}$, and then used for Transwell invasion assay. (D) HCT116 cells were transfected with cDNA-PDGF-D-lentivirus or negative control for $48 \mathrm{~h}$, and then used for Cell Counting kit- 8 assay. (E) The apoptotic rate of the negative control group was $9.1 \%$ by Annexin V/PI staining. (F) The apoptotic rate of the cDNA-PDGF-D-lentivirus group was $8.6 \%$ by Annexin V/PI staining. "P<0.05. V, vector plasmid alone; P, plasmid with PDGF-D clone; PDGF-D, platelet-derived growth factor-D; MMP-9, matrix metalloproteinase-9; PI, propidium iodide; OD, optical density; cDNA, complementary DNA; mRNA, messenger RNA; Q, quadrant; APC, allophycocyanin; SSC, side scatter.

cell line was successfully established by lentiviral transfection (Fig. 4A). Compared with that of the control HCT116 cells, the capacity of migration, invasion and proliferation of PDGF-D upregulated HCT116 cells were identified to be markedly increased (Fig. 4B-D). Our previous study results revealed that Notch1 and MMP-9 are associated with the migration, invasion and proliferation ability of colon cancer cells; thus, the expression of Notch1 and MMP-9 was detected in the PDGF-D-upregulated HTC116 cells. Notably, a significantly increased level of Notch1 and MMP-9 expression was observed in the PDGF-D-upregulated cells (Fig. 4A). Furthermore, the apoptotic rate of transfected HCT116 cells was detected. The results revealed that there was no clear difference between the cDNA-PDGF-D-lentivirus-transfected group and the negative control group (Fig. $4 \mathrm{E}$ and F; negative control vs. cDNA-PDGF-D-lentivirus=9.1 vs. $8.6 \%$ ). 


\section{Discussion}

CRC is the one of the most common types of malignancy around the world, with $>1.35$ million novel cancer cases and $\sim 690,000$ cancer-associated mortalities in 2012 (19). It has been widely accepted that tumorigenesis and tumor progression of CRC are associated with multiple epigenetic changes and molecular alterations $(20,21)$. Identifying more novel biomarkers and their corresponding molecular mechanism of carcinogenesis facilitates the development of new approaches for the prevention and treatment of CRC (22).

Several previous studies indicated that the expression of PDGF-D is upregulated in various malignancies, including prostate, breast, pancreatic and gastric cancer $(7-9,11)$. Furthermore, PDGF-D has been shown to be a critical factor that regulates the processes of cell proliferation, apoptosis, migration, invasion, angiogenesis and metastasis (23). As there were few studies concerning the function and mechanism of PDGF-D in CRC tumorigenesis and progression, the present study was performed.

According to the present study, 53.3\% (24/45) of CRC tissues exhibited overexpression of PDGF-D protein by immunohistochemical detection, and similarly, the majority of the CRC tissues exhibited overexpression of PDGF-D mRNA by $q \mathrm{PCR}$ assay, with a significant difference $(\mathrm{P}<0.05)$. These results revealed that $\mathrm{PDGF}-\mathrm{D}$ is one of the biomarkers that promotes the process of CRC oncogenesis, similar to other types of cancer reported previously $(24,25)$.

In order to unravel the potential mechanism, subsequent experiments were conducted in vitro. Human colon cell lines, including SW480, SW620, HCT116, CT26, DLD-1 and LoVo, were screened for the expression of PDGF-D (data of SW620, DLD-1 and LoVo cells are not shown). Finally, SW480 was selected for its overexpression of PDGF-D, and HCT116 was selected for its low expression of PDGF-D. In the two cell lines, the expression of PDGFR- $\beta$ exhibited similar changes, predicting that PDGF-D exerts its cellular functions through PDGFR- $\beta$. PDGF-D expression was then successfully downregulated in SW480 cells and upregulated in HCT116 cells. For the purpose of excluding the possibility that the following experiments were affected by different apoptosis rates subsequent to transfection, detection of apoptosis was conducted by flow cytometry. The results indicated that there was no clear difference between the experimental and negative control groups. As expected, the potential of migration, invasion and proliferation of cancer cells changed accordingly. These results suggest that PDGF-D promotes $\mathrm{CRC}$ tumorigenesis and progression by regulating the capacity of migration, invasion and proliferation in CRC.

Accumulating evidence suggests that PDGF-D promotes tumorigenesis and cancer progression by regulating several downstream signaling pathways (26-28). In prostate cancer, upregulated PDGF-D promotes cell proliferation and tumor growth through the mTOR signaling pathway (by activating the downstream targets S6 kinase and 4E binding protein) and upregulation of Bcl-2 (26). In breast cancer, overexpression of PDGF-D improves the aggression of cancer cells by upregulating the Notch, NF- $\kappa \mathrm{B}$ and CXCR4 signaling pathways $(17,27)$. In pancreatic cancer, overexpressed PDGF-D increases the capacity of proliferation and invasion by activating the Notch and NF- $\mathrm{B}$ signaling pathways (28). In the present study, it was shown that the expression of Notch1 and MMP-9 markedly decreased when PDGF-D was successfully downregulated in SW480 cells, and by contrast, the expression of Notch1 and MMP-9 increased significantly in HCT116 cells when PDGF-D was effectively upregulated. Since MMP-9 is the known downstream target of the Notch and NF- $\mathrm{B}$ signaling pathways $(29,30)$, the results may predict that PDGF-D promotes CRC migration, invasion and proliferation by regulating the Notch and/or NF- $\kappa \mathrm{B}$ signaling pathways. Therefore, future studies will provide more evidence about the association between PDGF-D and the Notch and $\mathrm{NF}-\kappa \mathrm{B}$ signaling pathways, and subsequently elucidate the complete mechanism.

In summary, the overexpression of PDGF-D, existing in the majority of human CRC tissues, promotes CRC migration, invasion and proliferation. In vitro experiments demonstrated that Notch1 and MMP-9 were upregulated by PDGF-D, and the invasiveness of cancer cells was distinctly enhanced as PDGF-D was overexpressed. Thus, the PDGF-D gene may be developed into a novel therapeutic target of human CRC.

\section{Acknowledgements}

The present study was supported by the National Natural Science Foundation of China (grant nos. 81271199 and 81470789).

\section{References}

1. Siegel R, Desantis C and Jemal A: Colorectal cancer statistics, 2014. CA Cancer J Clin 64: 104-117, 2014.

2. Center MM, Jemal A, Smith RA and Ward E: Worldwide variations in colorectal cancer. CA Cancer J Clin 59: 366-378, 2009.

3. Center MM, Jemal A and Ward E: International trends in colorectal cancer incidence rates. Cancer Epidemiol Biomarkers Prev 18: 1688-1694, 2009.

4. Morán A, Ortega P, de Juan C, Fernández-Marcelo T, Frías C Sánchez-Pernaute A, Torres AJ, Díaz-Rubio E, Iniesta P and Benito M: Differential colorectal carcinogenesis: Molecular basis and clinical relevance. World $\mathrm{J}$ Gastrointest Oncol 2: 151-158, 2010.

5. Andrae J, Gallini R and Betsholtz C: Role of platelet-derived growth factors in physiology and medicine. Genes Dev 22: 1276-1312, 2008

6. Li X and Eriksson U: Novel PDGF family members: PDGF-C and PDGF-D. Cytokine Growth Factor Rev 14: 91-98, 2003.

7. Kong D, Banerjee S, Ahmad A, Li Y, Wang Z, Sethi S and Sarkar FH: Epithelial to mesenchymal transition is mechanistically linked with stem cell signatures in prostate cancer cells. PLoS One 5: e12445, 2010.

8. Sethi S, Sarkar FH, Ahmed Q, Bandyopadhyay S, Nahleh ZA, Semaan A, Sakr W, Munkarah A and Ali-Fehmi R: Molecular markers of epithelial-to-mesenchymal transition are associated with tumor aggressiveness in breast carcinoma. Transl Oncol 4: 222-226, 2011.

9. Wang Z, Ali S, Banerjee S, Bao B, Li Y, Azmi AS, Korc M and Sarkar FH: Activated K-Ras and INK4a/Arf deficiency promote aggressiveness of pancreatic cancer by induction of EMT consistent with cancer stem cell phenotype. J Cell Physiol 228: 556-562, 2013.

10. Wu Q, Hou X, Xia J, Qian X, Miele L, Sarkar FH and Wang Z: Emerging roles of PDGF-D in EMT progression during tumorigenesis. Cancer Treat Rev 39: 640-646, 2013.

11. Zhao L, Zhang C, Liao G and Long J: RNAi-mediated inhibition of PDGF-D leads to decreased cell growth, invasion and angiogenesis in the SGC-7901 gastric cancer xenograft model. Cancer Biol Ther 9: 42-48, 2010.

12. Schläfli AM, Berezowska S, Adams O, Langer R and Tschan MP: Reliable LC3 and p62 autophagy marker detection in formalin fixed paraffin embedded human tissue by immunohistochemistry. Eur J Histochem 59: 2481, 2015. 
13. Jackson MF, Hoversten KE, Powers JM, Trobridge GD and Rodgers BD: Genetic manipulation of myoblasts and a novel primary myosatellite cell culture system: Comparing and optimizing approaches. FEBS J 280: 827-839, 2013.

14. Yan LH, Wei WY, Cao WL, Zhang XS, Xie YB and Xiao Q: Overexpression of E2F1 in human gastric carcinoma is involved in anti-cancer drug resistance. BMC Cancer 14: 904, 2014.

15. Bustin SA, Benes V, Garson JA, Hellemans J, Huggett J, Kubista M, Mueller R, Nolan T, Pfaffl MW, Shipley GL, et al: The MIQE guidelines: Minimum information for publication of quantitative real-time PCR experiments. Clin Chem 55: 611-622, 2009.

16. Livak KJ and Schmittgen TD: Analysis of relative gene expression data using real-time quantitative PCR and the 2(-Delta Delta C(T)) method. Methods 25: 402-408, 25, 2001.

17. Ahmad A, Wang Z, Kong D, Ali R, Ali S, Banerjee S and Sarkar FH: Platelet-derived growth factor-D contributes to aggressiveness of breast cancer cells by up-regulating Notch and NF- $\mathrm{KB}$ signaling pathways. Breast Cancer Res Treat 126: 15-25, 2011.

18. Li H, Tang J, Lei H, Cai P, Zhu H, Li B, Xu X, Xia Y and Tang W: Decreased MiR-200a/141 suppress cell migration and proliferation by targeting PTEN in hirschsprung's disease. Cell Physiol Biochem 34: 543-553, 2014.

19. Torre LA, Bray F, Siegel RL, Ferlay J, Lortet-Tieulent J and Jemal A: Global cancer statistics, 2012. CA Cancer J Clin 65: 87-108, 2015.

20. Stoffel EM: Screening in GI cancers: The role of genetics. J Clin Oncol 33: 1721-1728, 2015

21. Grady WM and Markowitz SD: The molecular pathogenesis of colorectal cancer and its potential application to colorectal cancer screening. Dig Dis Sci 60: 762-772, 2015

22. Tsujii M: Search for novel target molecules for the effective treatment or prevention of colorectal cancer. Digestion 85: 99-102, 2012.

23. Wang Z, Ahmad A, Li Y, Kong D, Azmi AS, Banerjee S and Sarkar FH: Emerging roles of PDGF-D signaling pathway in tumor development and progression. Biochim Biophys Acta 1806: $122-130,2010$
24. Kong D, Banerjee S, Huang W, Li Y, Wang Z, Kim HR and Sarkar FH: Mammalian target of rapamycin repression by 3,3'-diindolylmethane inhibits invasion and angiogenesis in platelet-derived growth factor-D-overexpressing PC3 cells. Cancer Res 68: 1927-1934, 2008

25. Wang Z, Kong D, Li Y and Sarkar FH: PDGF-D signaling: A novel target in cancer therapy. Curr Drug Targets 10: 38-41, 2009.

26. Kong D, Wang Z, Sarkar SH, Li Y, Banerjee S, Saliganan A, Kim HR, Cher ML and Sarkar FH: Platelet-derived growth factor-D overexpression contributes to epithelial-mesenchymal transition of PC3 prostate cancer cells. Stem Cells 26: 1425-1435, 2008.

27. Liu J, Liao S, Huang Y, Samuel R, Shi T, Naxerova K, Huang P, Kamoun W, Jain RK, Fukumura D and Xu L: PDGF-D improves drug delivery and efficacy via vascular normalization, but promotes lymphatic metastasis by activating CXCR4 in breast cancer. Clin Cancer Res 17: 3638-3648, 2011.

28. Wang Z, Kong D, Banerjee S, Li Y, Adsay NV, Abbruzzese J and Sarkar FH: Down-regulation of platelet-derived growth factor-D inhibits cell growth and angiogenesis through inactivation of Notch-1 and nuclear factor-kappaB signaling. Cancer Res 67: $11377-11385,2007$.

29. Ranganathan P, Weaver KL and Capobianco AJ: Notch signalling in solid tumours: A little bit of everything but not all the time. Nat Rev Cancer 11: 338-351, 2011.

30. Fraser CC: Exploring the positive and negative consequences of NF-kappaB inhibition for the treatment of human disease. Cell Cycle 5: 1160-1163, 2006

(i) $\ominus$ This work is licensed under a Creative Commons (c) ${ }_{\text {EY NO ND }}$ Attribution-NonCommercial-NoDerivatives 4.0 International (CC BY-NC-ND 4.0) License. 